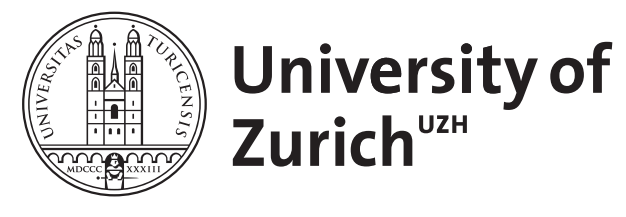

\title{
Isolated oculomotor nerve palsy related to sinusitis?
}

\author{
Sturm, V ; Kordic, H ; Leiba, H ; Landau, K
}

\begin{abstract}
The association of sinusitis with ocular motility disorders is a seductive theory due to their close anatomical vicinity. Typically, sinusitis can influence ocular motility by affecting single muscles or a combination of muscles and/or cranial nerves due to a local inflammatory tissue reaction. Although rare, sinusitis has been suggested at least as a cause for superior-branch oculomotor palsy. We report a case of progressive, isolated, third-cranial-nerve palsy caused by an aneurysm of the posterior-communicating artery that initially was thought to be related to pansinusitis.
\end{abstract}

DOI: https://doi.org/10.1007/s10792-007-9132-z

Posted at the Zurich Open Repository and Archive, University of Zurich ZORA URL: https://doi.org/10.5167/uzh-8186

Journal Article

Published Version

Originally published at:

Sturm, V; Kordic, H; Leiba, H; Landau, K (2008). Isolated oculomotor nerve palsy related to sinusitis? International Ophthalmology, 28(4):303-305.

DOI: https://doi.org/10.1007/s10792-007-9132-z 


\title{
Isolated oculomotor nerve palsy related to sinusitis?
}

\author{
Veit Sturm • Helena Kordic · Hana Leiba • \\ Klara Landau
}

Received: 24 April 2007 / Accepted: 8 August 2007/Published online: 5 September 2007

(C) Springer Science+Business Media B.V. 2007

\begin{abstract}
The association of sinusitis with ocular motility disorders is a seductive theory due to their close anatomical vicinity. Typically, sinusitis can influence ocular motility by affecting single muscles or a combination of muscles and/or cranial nerves due to a local inflammatory tissue reaction. Although rare, sinusitis has been suggested at least as a cause for superior-branch oculomotor palsy. We report a case of progressive, isolated, third-cranial-nerve palsy caused by an aneurysm of the posteriorcommunicating artery that initially was thought to be related to pansinusitis.
\end{abstract}

Keywords Oculomotor nerve palsy .

Sinusitis · Intracranial aneurysm $\cdot$ Pupil-involving

\section{Introduction}

Most cases of isolated oculomotor nerve palsy are related to ischemia or aneurysm or are the consequence of a tumor or head trauma. Many other

V. Sturm $(\bowtie) \cdot$ H. Kordic $\cdot$ K. Landau

Department of Ophthalmology,

University Hospital of Zurich,

Frauenklinikstrasse 24, 8091 Zurich, Switzerland

e-mail: veit.sturm@usz.ch

H. Leiba

Department of Ophthalmology, Kaplan Medical Center,

Rehovot, Israel etiologies had been suggested; however, the cause in almost every fourth case remains uncertain [1]. A relationship between isolated third-nerve palsy and sinusitis seems enticing due to their close anatomical vicinity. Although rare, sinusitis has been suggested as a cause for superior branch oculomotor palsy [2]. A delayed diagnosis of a compressive intracranial aneurysm in conjunction with sinusitis has also been reported [3]. We report a case of progressive, isolated, third-cranial-nerve palsy caused by an aneurysm of the posterior-communicating artery that initially was thought to be related to pansinusitis.

\section{Case report}

A 39-year-old previously healthy woman presented with fever and vomiting. Four weeks earlier, she started to suffer from a cold and right-sided head and facial pain accompanied with ipsilateral painful eye movements and photophobia. Computed tomography demonstrated opacification of the right sphenoid and ethmoid sinus as well as the left maxillary sinus (Figs. 1,2). A major involvement of the orbital apex could be ruled out. She was treated with intravenous administration of broad-spectrum antibiotics for 1 week because of the diagnosis of a pansinusitis.

Magnetic resonance imaging (MRI) 5 days later did not show a considerably larger expansion but confirmed the diagnosis of sinusitis. The patient continued to suffer from painful eye movements on 


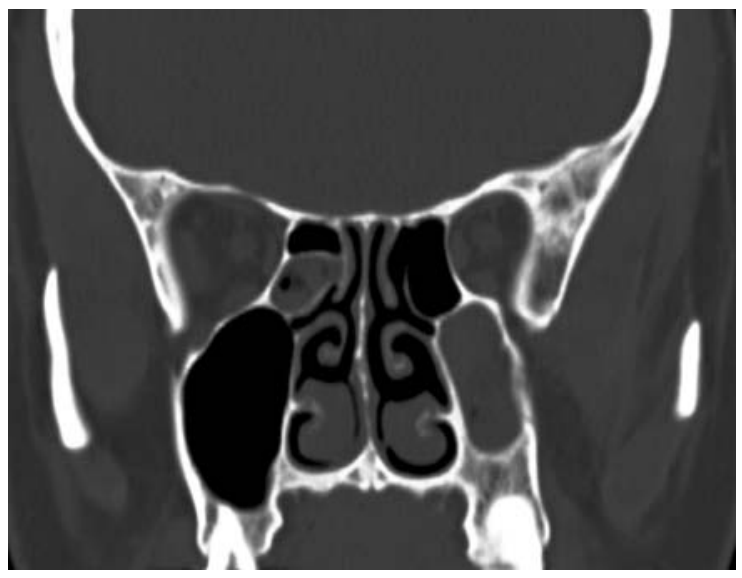

Fig. 1 Initial computed tomography demonstrating opacification of the right ethmoid sinus and the left maxillary sinus

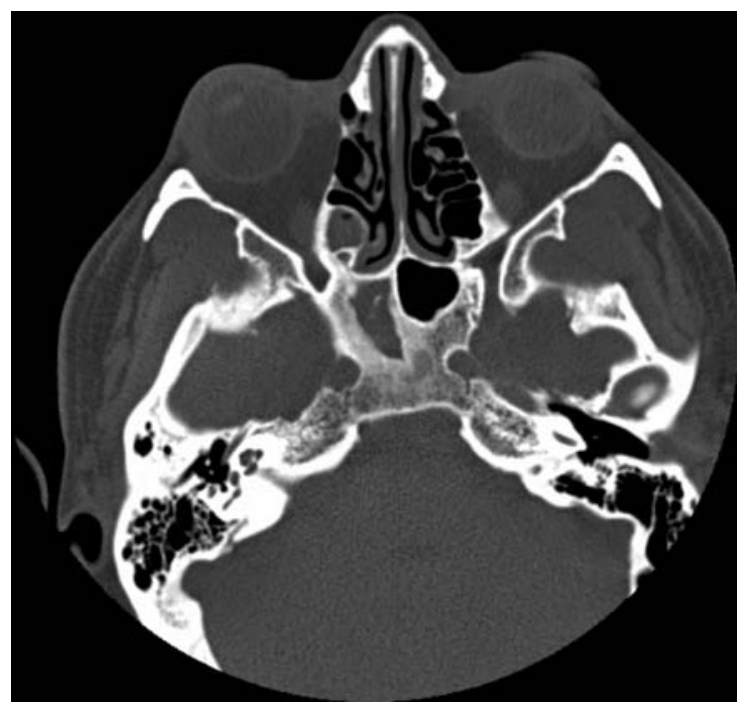

Fig. 2 Initial computed tomography demonstrating opacification of the right ethmoid sinus and the right sphenoid sinus

the right and complained of increasing right-sided head pain. Flu-like symptoms and difficulty in breathing worsened, but an ophthalmological examination 2 days later revealed no ocular abnormalities. Two days later, the patient developed right-side ptosis and dilated pupil. A diagnosis of pupilinvolving third-nerve palsy due to progressive sinusitis and presumed extension of the orbital apex was made, and the patient underwent a sinus surgery. A repeated MRI 3 weeks later revealed mild improvement of the sinusitis. Despite the surgery and continuous antibiotic treatment, no striking clinical improvement was noticed, and she experienced two other major pain episodes. The patient was transferred to our institution for further evaluation and treatment.

On admission, examination confirmed pupilinvolving oculomotor nerve palsy (Fig. 3). Cerebral catheter angiography revealed a large aneurysm of the right posterior-communicating artery and a smaller one of the right medial cerebral artery bifurcation (Fig. 4). Clipping of both aneurysmal necks was performed.

\section{Discussion}

Our patient suffered from an isolated, pupil-involving, third-cranial-nerve palsy due to a life-threatening posterior-communicating artery aneurysm. In retrospect, the initial photophobia may have indicated early pupil involvement, and the severe pain episodes could be interpreted as an alarm signal of a threatening aneurysm rupture. The "close-lying" connections, as seductive they are, should not divert from the rule: infrequent causes are infrequent and frequent causes are frequent! A third-cranial-nerve palsy in association with sinusitis without further cranial-nerve participation or severe orbital involvement should be considered in the diagnosis, even though exceedingly rare, in light of the anatomical course with its final dividing in a superior and inferior branch. Therefore, patients with an isolated pupil-involving oculomotor nerve palsy should undergo catheter angiography or magnetic resonance angiography, even if several

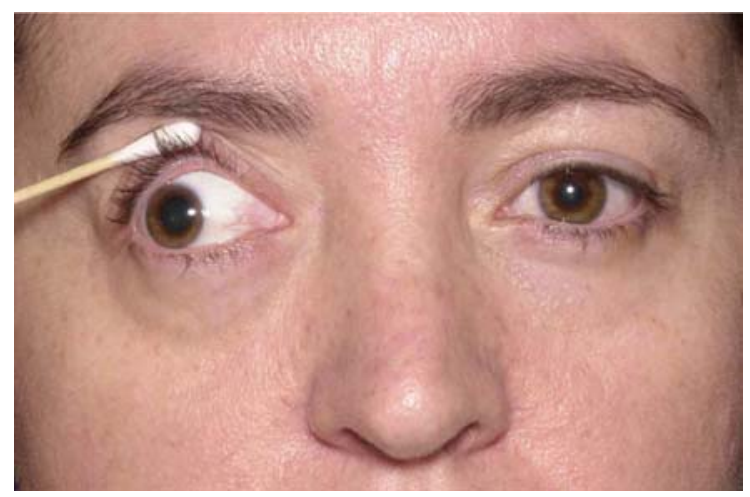

Fig. 3 Photograph of patient on admission to our institution. A pupil-involving oculomotor nerve palsy was present 
Fig. 4 Cerebral catheter angiography showing large aneurysm of the right posterior-communicating artery (left, arrow) and a smaller one of the right medial cerebral artery bifurcation (right, arrow)

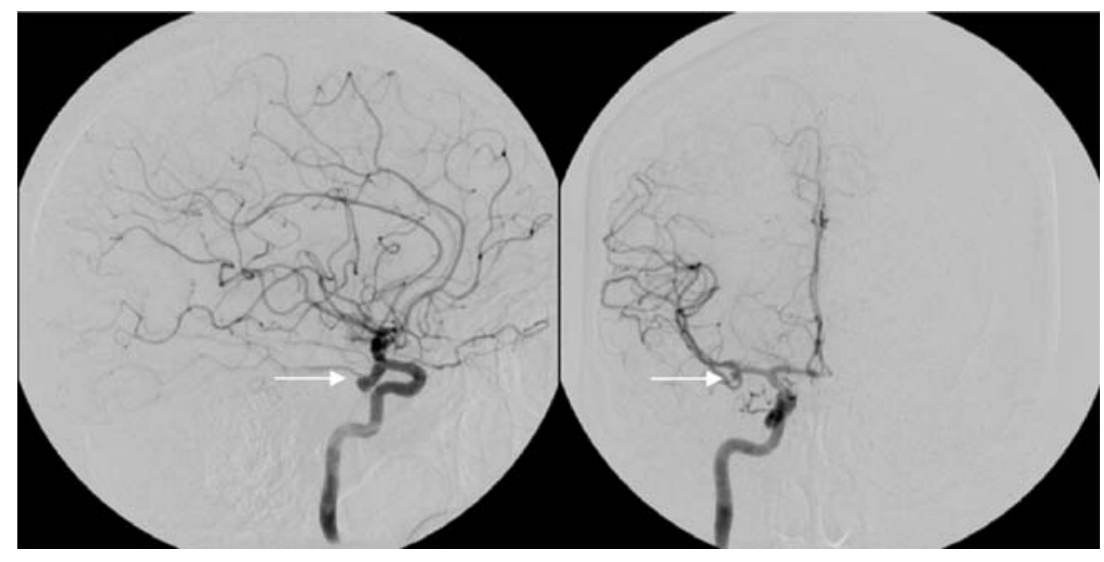

imagings do not reveal an aneurysm. Detection of a cerebral aneurysm could be lifesaving [4].

\section{References}

1. Richards BW, Jones R, Younge BR (1992) Causes and prognosis in 4278 cases of paralysis of the oculomotor, trochlear and abducens cranial nerves. Am J Ophthalmol 113:489-496
2. Stefanis L, Przedborski S (1993) Isolated palsy of the superior branch of the oculomotor nerve due to chronic erosive sphenoid sinusitis. J Clin Neuro-ophthalmol 13(4): 229-231

3. Timchenko V, Cramer B, Kaden B, Kempf HG (2002) Einseitige Ptosis - orbitale Komplikation? HNO 50: 490-491

4. Trobe JD (1998) Managing oculomotor nerve palsy. Arch Ophthalmol 116:798 